Research Paper

\title{
Expression of Polo-Like Kinase 4(PLK4) in Breast Cancer and Its Response to Taxane-Based Neoadjuvant Chemotherapy
}

\author{
Zhenhua Li*, Kun Dai*, Chijuan Wang, Yawen Song, Feng Gu, Fangfang Liu ${ }^{凶}, \mathrm{Li} \mathrm{Fu}^{凶}$ \\ Department of Breast Pathology and Research Laboratory, Key Laboratory of Breast Cancer Prevention and Therapy (Ministry of Education), National Clinical \\ Research Center for Cancer, Tianjin Medical University Cancer Institute and Hospital, Huanhuxi Road, Tianjin 300060, China. \\ *These authors contributed equally to this work. \\ $\triangle$ Corresponding authors: Li Fu and Fangfang Liu, Postal address: Huanhuxi Road, Tiyuanbei, Hexi District, Tianjin300060, China. E-mail: \\ fulijyb@hotmail.com; lef527@163.com Telephone Number: +86-22-23340123-6006 Fax Number: +86-22-23340123-6019. \\ ( ) Ivyspring International Publisher. Reproduction is permitted for personal, noncommercial use, provided that the article is in whole, unmodified, and properly cited. See \\ http://ivyspring.com/terms for terms and conditions.
}

Received: 2015.11.03; Accepted: 2016.03.22; Published: 2016.06.06

\begin{abstract}
Purpose: Polo-like kinase 4(PLK4) is an important evolutionarily regulator involved in centrosome duplication. We here investigated the expression of PLK4 mRNA and PLK4 in breast cancer, and evaluated its predictive value for response to taxane-based neoadjuvant chemotherapy.

Method: The PLK4 mRNA expression was measured in breast cancer tissues and corresponding normal breast tissues from 30 breast cancer patients by quantitative real-time polymerase chain reaction (PCR).The association of the expression of PLK4 with clinicopathological parameters and prognostic significance was evaluated in 154 cases of invasive breast cancer. In addition, we immunohistochemically examined the changes of PLK4 expression in biopsy and postoperative tumor specimens of another 64 breast cancer patients who received taxane-based neoadjuvant chemotherapy.

Results: The level of PLK4 mRNA expression in cancerous tissues had a significant difference compared to the corresponding normal breast tissues $(P=0.021)$. There is a correlation of PLK4 expression with higher incidence of lymph node metastasis and distant metastasis or surrounding recurrence $(P=0.043 ; P=0.006)$. High $P L K 4$ expression was found to be a detrimental prognostic factor measured by overall survival (OS) $(P=0.003)$ and progress-free survival (PFS) $(P=0.003)$. Moreover, the results demonstrated that PLK4 expression was a negative predictor of response to taxane-based neoadjuvant chemotherapy $\left(r_{s}=-0.253, P=0.044\right)$.

Conclusion: The findings of this current study indicated that PLK4 expression in breast cancer could be a potential prognostic factor and a negative predictor of response to taxane-based neoadjuvant chemotherapy.
\end{abstract}

Key words: Polo-like kinase 4 (PLK4), Breast cancer, Neoadjuvant chemotherapy, Survival, Resistance.

\section{Introduction}

Polo-like kinase 4(PLK4), an important evolutionarily regulator involved in centrosomes duplication, is structurally distinguished from other members of polo-like kinase family [1]. Overexpression of PLK4 can induce centrosome amplification and chromosome instability [2], which are hallmarks of cancer cells, and contribute to tumor aggressiveness $[3,4]$. Given the fact that the features frequently presented in cancers, some studies have devoted to demonstrating the mitotic kinase activities in human tumors, including colorectal, gastric, and hepatocellular carcinoma $[2,5,6]$. However, no studies 
of PLK4 expression and resultant activities have been reported in breast cancer.

Neoadjuvant chemotherapy for breast cancer is a supplement therapeutic modality that can reduce the tumor sizes, reduce node and distant micro-metastases, and improve operability [7]. The taxane-based chemotherapy is commonly used [8,9], for example taxane and anthracycline. However, the regimen just has benefit to a portion of patient population, affecting their overall clinical outcome [10]. Taxanes can kill cancer cells or inhibit the proliferation of cancer cells through interfering with microtubule functions. Moritz and Agard reported that $\gamma$-Tubulin is thought to be responsible for microtubule nucleation by forming a multiprotein ring complex ( $\gamma$-TuRC) [11], and PLK4 is an upstream regulator for $\gamma$-tubulin, which is dependent on format of the $\gamma$-tubulin-containing structure [12]. Thus, it is conjectured that PLK4 is possibly involved in chemo-resistance.

The aim of this current study was to investigate PLK4 expression in breast cancer and to evaluate its predictive value for response totaxane-based neoadjuvant chemotherapy. Therefore, we examined whether PLK4 aberrantly expressed in breast cancer by immunohistochemistry and, and evaluated the relationship of PLK4expression with the response totaxane-based neoadjuvant chemotherapy.

\section{Materials and methods}

\section{Specimen selection and clinical information}

In this study, we selected randomly 154 cases of invasive breast cancer patients who were diagnosed from January 2004 to December 2009 were retrieved from the archive of Tianjin University Cancer Hosptial (Tianjin, China). The diagnose of all cases and lymph node metastasis were evaluated in $4 \mu \mathrm{m}$ sections, stained hematoxyline and eosin, by two experienced pathologists (Fangfang $\mathrm{Liu}, \mathrm{Li} \mathrm{Fu}$ ). Histologic grade was assessed according to the Bloom and Richardson grading system [13]. The clinicopathological information of patients is showed in Table 1.

In addition, we selected another 64 patients hospitalized from October 2005 to December 2010, who were diagnosed with invasive breast cancer by 14-gauge core needle biopsy and completed the taxane-basedneoadjuvant chemotherapy (consisted of 2 to 8 cycles of TE) combining with chemotherapy regimen without local or systemic treatment before surgery. The specimens were collected from each patient's core needle biopsy of primary breast tumor before neoadjuvant chemotherapy and corresponding matching postoperative tumor tissues.
Table 1. Correlation of PLK4 expression with clinicopathological parameters of breast cancer.

\begin{tabular}{|c|c|c|c|c|}
\hline \multirow[t]{2}{*}{ Characteristic } & \multicolumn{2}{|c|}{ PLK4expression[n(\%)] } & \multirow[t]{2}{*}{$r_{s}$} & \multirow[t]{2}{*}{$P$} \\
\hline & Low & High & & \\
\hline No.of patients & $77(50.0)$ & $77(50.0)$ & & \\
\hline Age(years) & & & 0.052 & 0.522 \\
\hline$<50$ & $41(53.2)$ & $37(48.1)$ & & \\
\hline$\geq 50$ & $36(46.8)$ & $40(51.9)$ & & \\
\hline Tumor size(cm) & & & 0.031 & 0.706 \\
\hline$\leq 2$ & $19(24.7)$ & $17(22.1)$ & & \\
\hline$>2$ & $58(75.3)$ & $60(77.9)$ & & \\
\hline Lymph node & & & 0.163 & 0.043 \\
\hline Negative & $33(42.9)$ & $21(27.3)$ & & \\
\hline Positive & $44(57.1)$ & $56(72.7)$ & & \\
\hline Metastasis, recurrence & & & 0.220 & 0.006 \\
\hline Negative & $64(83.1)$ & $49(63.7)$ & & \\
\hline Positive & 13(16.9) & $28(36.3)$ & & \\
\hline Histological grade $\neq$ & & & 0.120 & 0.163 \\
\hline I & $6(7.8)$ & $4(5.2)$ & & \\
\hline II & $53(68.8)$ & $48(62.3)$ & & \\
\hline III & 10(13.0) & $16(20.8)$ & & \\
\hline ER & & & -0.092 & 0.256 \\
\hline Negative & $29(37.7)$ & $36(46.8)$ & & \\
\hline Positive & $48(63.3)$ & $41(53.2)$ & & \\
\hline PR & & & -0.066 & 0.416 \\
\hline Negative & $29(37.7)$ & $34(44.2)$ & & \\
\hline Positive & $48(63.3)$ & $43(55.8)$ & & \\
\hline HER2 & & & 0.084 & 0.300 \\
\hline Negative & $56(72.7)$ & $50(64.9)$ & & \\
\hline Positive & $21(27.3)$ & $27(35.1)$ & & \\
\hline Molecular subtypes & & & 0.043 & 0.595 \\
\hline Luminal- & $54(70.1)$ & $50(64.9)$ & & \\
\hline HER-2-enriched & $7(9.1)$ & $11(14.3)$ & & \\
\hline Basal-like & $16(20.8)$ & $16(20.8)$ & & \\
\hline Chemotherapy $\$$ & & & -0.093 & 0.266 \\
\hline Taxane-based & 24(31.1) & $29(37.7)$ & & \\
\hline No-taxane-based & $50(64.9)$ & $41(53.2)$ & & \\
\hline
\end{tabular}

Molecular subtypes of the tumors were authenticated through additional immunohistochemistry performed on serial tissuesections using the standard procedures according to our previous studies $[14,15]$.

\section{Tatal RNA samples, reverse transcription and quantitative real-time polymerase chain reaction (PCR)}

Breast cancer tissues and corresponding normal breast tissues from breast cancer patients $(n=30)$ were obtained from Tianjin University Cancer Hosptial (China). The total RNA was extracted using RNeasy Plus Mini Kit (Qiagen, Valencia, USA) and converted to cDNA using PrimeScript RT Master Mix Kit (Takara, Otsu, Japan) following the manufacturer's protocol. The quantitative real-time PCR was performed with an Bio-Rad CFX96 real-time PCR system using SYBR Premix Ex Taq IIKit (Takara, Otsu, Japan) with the following thermal cycle protocol: (1) $95^{\circ} \mathrm{C}$ for $30 \mathrm{~s}$; (2) $95^{\circ} \mathrm{C}$ for $5 \mathrm{~s}, 40 \times$; (3) $60^{\circ} \mathrm{C}$ for $34 \mathrm{~s}, 40 \times$. The following PCR primers were used: $5^{\prime}$ - CCT TAT 
CAC CTC CTC CTT C-3' and 5' - CCA AGT CCT TCA TTT GTA ACC-3' for PLK4 and 5'- ACA TCA TCC CTG CCT CTA C-3' and 5'- CCT GCT TCA CCA CCT TCT-3' for GAPDH. The relative amounts of PLK4 transcript were normalized to those of the GAPDH transcript. The $\mathrm{T} / \mathrm{N}$ values were measured by dividing the normalized transcript amounts in breast cancer tissues by which in corresponding normal breast tissues.

\section{Immunohistochemistry and quantification}

Immunohistochemical staining was performed using standard procedures. Briefly, $4 \mu \mathrm{m}$ tissue sections were dewaxed and subsequently rehydrated using xylene and graded alcohol washes.Antigen retrieval was performed at $121{ }^{\circ} \mathrm{C}$ for $2 \mathrm{~min}$, using citrate buffer $(\mathrm{pH}$ 6.0). After serial blocking with hydrogen peroxide and normal goat serum, the sections were incubated with primary polyclonal antibody against PLK4 (Abcam, ab137398, polyclonal, 1:100 dilution) for $16 \mathrm{~h}$ at $4{ }^{\circ} \mathrm{C}$. Then, the sections were sequentially incubated with biotinylated goat anti-mouse immunoglobulin and peroxidaseconjugated streptavidin.

The stained PLK4 was found in the cytoplasm of tumor cells (Fig.1), hence, cells were considered positive for PLK4 when a clear-cut brown staining was observed in cytoplasm. Visual evaluation was conducted for estimating the percentage of positive tumuor cells and the intensity of staining. The intensity of staining was graded as follow: 0, no staining; 1, light staining; 2, moderate staining; 3 , strong staining. The final score was histological score

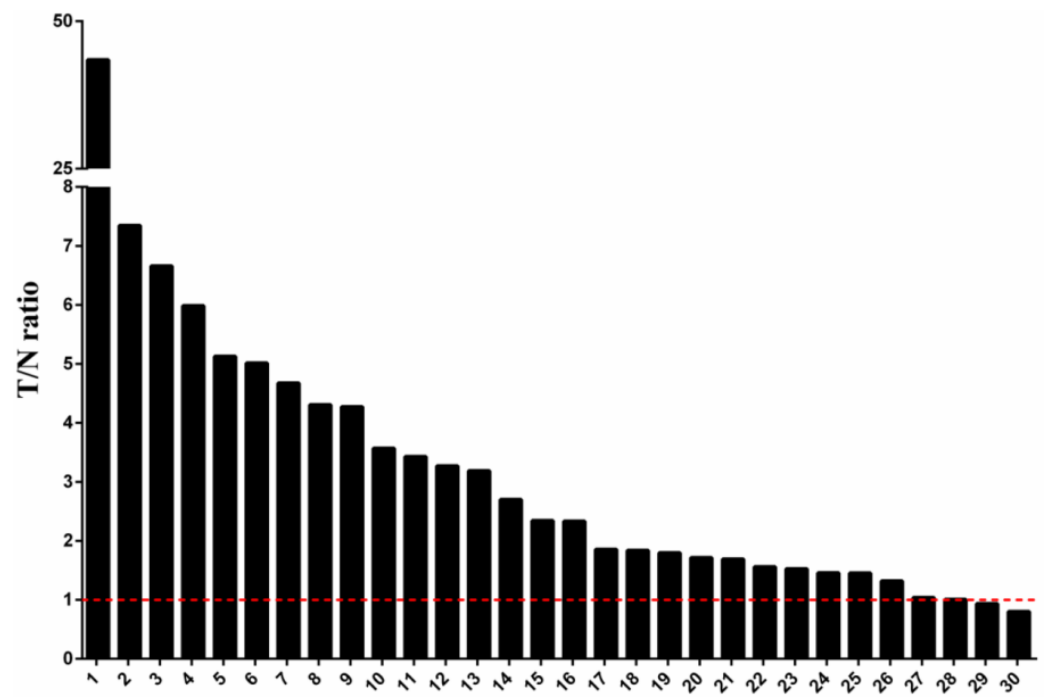

Figure 1. Upregulation of PLK4 mRNA expression level in human breast cancer. The mRNA expression level of PLK4 was compared in cancerous tissues and corresponding normal breast tissues from 30 breast cancer patients using a quantitative real-time PCR. After normalizing the amounts of PLK4 transcripts to those of the GAPDH transcripts, the T/N values were measured by dividing the normalized transcript amounts in breast cancer tissues by which in corresponding normal breast tissues.
(H-score), which is characterized by multiplying the percentage of positive tumor cells by the intensity of staining. The $\mathrm{H}$-score $\leq$ median was regarded as low expression, otherwise was regarded as high expression.

\section{Assessing of theresponse to neoadjuvant chemotherapy}

According to Miller and Payne histologic grading system[16], the response to chemotherapy was classified into five grades: grade 1 , no change or some alteration to individual malignant cells but no reduction in overall cellularity; grade 2, minor loss (up to $30 \%$ ) of cancer cells but overall cellularity remains high; grade 3, reduction of $30 \%$ to $90 \%$ of cancer cells; grade 4, more than $90 \%$ loss of cancer cells but small clusters or widely dispersed individual cancer cells remains; grade 5, no malignant cells identifiable in sections from the site of the tumor consisting of vascular fibroblastic stroma, often containing macrophages; however, ductal carcinoma in situ (DCIS) may be present. In this study, grade 1 was regarded as having no response to chemotherapy, grades 2 and 3 were regarded as having partial response, and grades 4 and 5 were regarded as having good response.

\section{Statistical analysis}

Statistical analysis was performed with SPSS 19.0 software (SPSS, IBM). Wilcoxon matched pairs test was used to detect the difference of PLK4 expression level between the cancerous tissues and the corresponding normal breast tissues. The survival curves (PFS, OS) were drawn using Kaplan-Meier estimates and compared using log-rank tests. Significant prognostic factors were found by using univariate and multivariate Cox-regression analysis. Spearman's Rank-Correlation test was performed to demonstrate the association between PLK4 expression and response to taxane-based neoadjuvant chemotherapy. $X^{2}$ test was used to evaluate the changes of PLK4 expression before and after neoadjuvant chemotherapy. A two-side $P<0.05$ was considered as an indicative of statistical difference.

\section{Results}

\section{Upregulation of PLK4 mRNA expression in human breast cancer}

We examined the expression of PLK4 mRNA in 30 breast cancer patients 
and calculated the ratio of the level of PLK4 mRNA expression in cancerous tissues to the level in the corresponding normal breast tissues $(\mathrm{T} / \mathrm{N}$ ratio). In Fig.1, we could clearly find that the expression of PLK4 mRNA was upregulated in $26(86.7 \%)$ of the 30 patients, and only 2 cases $(6.7 \%)$ showed downregulation of PLK4 mRNA expression. Moreover, the PLK4 expression level in cancerous tissues had a significant difference compared to the corresponding normal breast tissues $(P=0.021)$.

\section{The association of PLK4 expression with clinicopathological parameters}

The PLK4 expression in 154 breast cancer samples was examined by immunohistochemical staining. The samples were divided into four groups based on the staining intensity and the percentage (Fig. 2A). It is clear that only $2.6 \%$ of samples showed no expression of PLK4 (Fig. 2B).
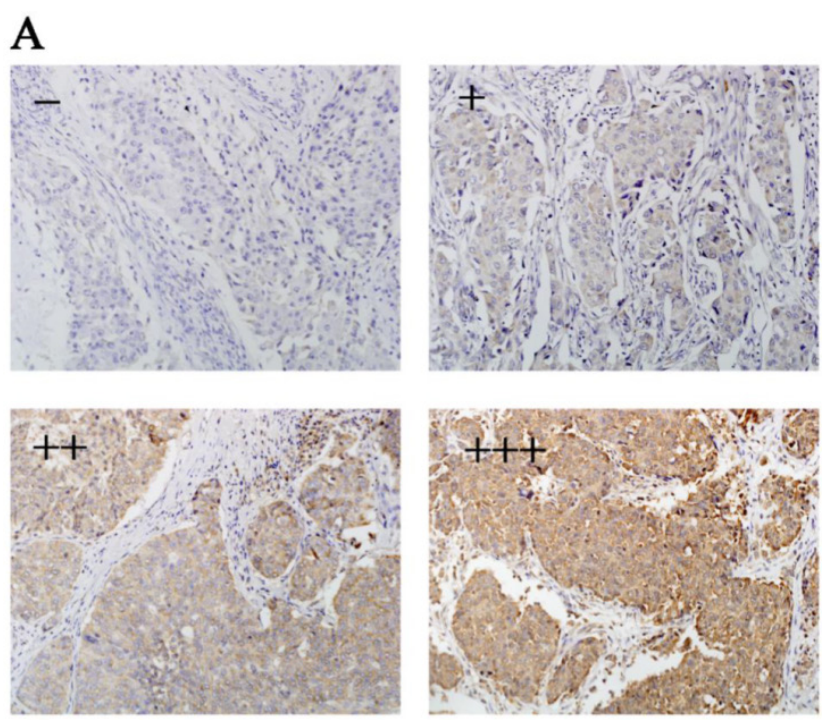

B

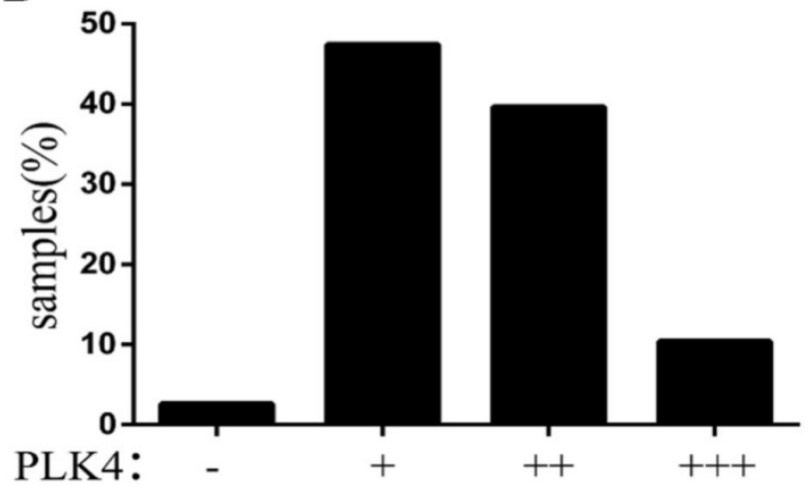

Figure 2. PLK4 expression in invasive breast cancer. A: the specimens were divided into four groups according to the staining intensity and the percentage; B: the percentage of the four groups.
In order to study the correlation of PLK4 expression with clinical characteristics in breast cancer, several clinicopathological parameters were analyzed. The results showed that PLK4 expression had a correlation with higher incidence of lymph node metastasis and distant metastasis, or surrounding recurrence $(\mathrm{P}=0.043 ; \mathrm{P}=0.006)$ (Table 1; Fig.3A and $3 \mathrm{~B})$. However, no significant correlation was found between PLK4 and other parameters, including age, tumor size, histological grade, ER status, PR status, HER2 status, and molecular subtypes (Table 1 ).

\section{Prognostic significant of PLK4 expression in breast cancer tissue}

High PLK4 expression was found to be a detrimental prognostic factor measured by OS $(P=0.003)$ and PFS $(P=0.003)$ (Fig. 3C, 3D). Univariate Cox-regression analysis also confirmed that high expression of PLK4 was an unfavorable predictor of OS $(P=0.010)$ and PFS $(P=0.004)$, in addition to the demonstration of prognostic significance of lymph node status. Negative ER and PR status were unfavorable factors of OS $(P=0.012, P=0.035)$, but not of PFS $(P=0.179, P=0.668)$ (Table 2$)$.

Table 2. Univariate Cox-regression analysis for OS and PFS.

\begin{tabular}{|c|c|c|c|c|c|c|}
\hline Factors & & OS & & & PFS & \\
\hline & HR & $95 \% \mathrm{CI}$ & $\mathrm{P}$ & HR & $95 \% \mathrm{CI}$ & $\mathrm{P}$ \\
\hline Age $(<50$ vs. $\geq 50)$ & 0.581 & $0.195-1.735$ & 0.331 & 1.014 & $0.549-1.871$ & 0.965 \\
\hline $\begin{array}{l}\text { Tumor size, } \mathrm{cm} \\
(\leq 2 \text { vs. }>2)\end{array}$ & 0.688 & $0.215-2.195$ & 0.527 & 0.915 & $0.448-1.870$ & 0.808 \\
\hline $\begin{array}{l}\text { Tumor Grade } \\
\text { (Ivs. IIvs.III) }\end{array}$ & 1.674 & $0.547-5.128$ & 0.367 & 1.568 & $0.822-2.992$ & 0.172 \\
\hline $\begin{array}{l}\text { Node } \\
\text { statue }(\mathrm{cN}-v \text { s.cN }+)\end{array}$ & 9.381 & $1.223-71.931$ & 0.031 & 3.967 & $1.664-9.459$ & 0.002 \\
\hline ER status( - vs. + ) & 0.195 & $0.054-0.700$ & 0.012 & 0.657 & $0.356-1.212$ & 0.179 \\
\hline PR status( - vs. +) & 0.288 & $0.090-0.918$ & 0.035 & 0.874 & $0.471-1.620$ & 0.668 \\
\hline $\begin{array}{l}\text { HER-2 status( - } \\
\text { vs. +) }\end{array}$ & 0.368 & $0.082-1.646$ & 0.191 & 1.070 & $0.554-2.065$ & 0.841 \\
\hline $\begin{array}{l}\text { PLK4 (low vs. } \\
\text { high) }\end{array}$ & 7.171 & $1.602-32.092$ & 0.010 & 2.624 & $1.355-5.085$ & 0.004 \\
\hline
\end{tabular}

Multivariate Cox-regression analysis demonstrated that high PLK4 expression was an indicator for worse PFS $(P=0.007)$, while the association with OS was not proved to be significant $(P=0.088)$. In addition, it could be deduced from table 3 that Lymph node status is an unfavorable prognostic factor of PFS $(P=0.019)$, while ER and HER2 status were found in OS $(P=0.042, P=0.035)$.

The association between PLK4 expression and response to taxane-based neoadjuvant chemotherapy

It can be seen from table 4 that 21 patients showed a good response, 32 patients had a partial 
response, and 11 patients showed no response among all 64 patients who had completed with preoperative taxane-based neoadjuvant chemotherapy. Spearman's Rank-Correlation test was performed to examine the relationship between PLK4 expression and response to taxane-based neoadjuvant chemotherapy. The results showed that PLK4 expression was a negative predictor of response to taxane-based neoadjuvant chemotherapy $\left(r_{s}=-0.253, P=0.044\right)$.

\section{Changes of PLK4 expression before and after neoadjuvant chemotherapy}

Compared to PLK4 expression at pre-neoadjuvant chemotherapy, PLK4 expression was found have a significant change overall at post-neoadjuvant chemotherapy $(P=0.003)$ (Fig. 4A). Further analysis showed that there were no significant changes of PLK4 expression between the biopsy and tumor specimens of breast cancer patients who had no response to taxane-based neoadjuvant chemotherapy $(P=0.611)$, while there was a significant decrease in the patients with partial response or good response $(P=0.031, P=0.008)$ (Fig. 4B, 4C, 4D).

There was low expression of PLK4 in 33cases at pre-neoadjuvant chemotherapy, and only one case had a high expression after neoadjuvant chemotherapy. Compared to the cases with low expression of PLK4, the cases with high expression showed a significant decrease after neoadjuvant chemotherapy. In light of the changes of PLK4 expression after neoadjuvant chemotherapy, we

divided the cases with high PLK4 expression into two groups: decrease and invariability. Moreover, we demonstrated that percentage of cases, showing no response in the invariability group, was significantly higher than that in the decrease group, in contrast to the cases in partial or good response groups $(P=0.004)$. (Fig. 4E)

Table 3. Multivariate Cox-regression analysis for OS and PFS.

\begin{tabular}{|c|c|c|c|c|c|c|}
\hline Factors & OS & & & PFS & & \\
\hline & HR & $95 \% \mathrm{CI}$ & $P$ & HR & $95 \% \mathrm{CI}$ & $P$ \\
\hline $\operatorname{Age}(<50$ vs. $\geq 50)$ & 0.966 & $0.260-3.591$ & 0.958 & 1.223 & $0.603-2.479$ & 0.577 \\
\hline $\begin{array}{l}\text { Tumor size, } \mathrm{cm}(\leq 2 \\
\text { vs. }>2)\end{array}$ & 0.492 & $0.128-1.900$ & 0.304 & 0.744 & $0.364-1.649$ & 0.507 \\
\hline $\begin{array}{l}\text { Tumor Grade (Ivs. } \\
\text { IIvs.III) }\end{array}$ & 0.904 & $0.209-3.914$ & 0.893 & 1.344 & $0.670-2.698$ & 0.405 \\
\hline $\begin{array}{l}\text { Node } \\
\text { statue(cN-vs.cN }+ \text { ) }\end{array}$ & 5.779 & $0.616-54.212$ & 0.125 & 2.995 & $1.196-7.500$ & 0.019 \\
\hline ER status( - vs. +) & 0.089 & $0.009-0.921$ & 0.042 & 0.495 & $0.191-1.281$ & 0.147 \\
\hline PR status( - vs. +) & 1.080 & $0.131-8.885$ & 0.943 & 1.132 & $0.451-2.842$ & 0.792 \\
\hline $\begin{array}{l}\text { HER-2 status( - vs. } \\
+ \text { ) }\end{array}$ & 0.083 & $0.008-0.835$ & 0.035 & 0.541 & $0.245-1.193$ & 0.128 \\
\hline PLK4(low vs. high) & 4.903 & $0.791-30.414$ & 0.088 & 2.929 & $1.340-6.405$ & 0.007 \\
\hline
\end{tabular}

Table 4. The relationship between PLK4 expression and response to taxane-based neoadjuvant chemotherapy in 64 paired specimens.

\begin{tabular}{lllll}
\hline Groups & \multicolumn{2}{c}{ PLK4 expression(n) } & rs & P \\
\cline { 2 - 3 } & Low & High & & \\
\hline No response & 2 & 9 & -0.253 & 0.044 \\
Partial response & 18 & 14 & & \\
Good response & 13 & 8 & & \\
\hline
\end{tabular}

Note: Statistical analysis was used Spearman's Rank-Correlation test $(\mathrm{n}=64)$. Specimens were collected from each patient's core needle biopsy of primary breast tumor before neoadjuvant chemotherapy.

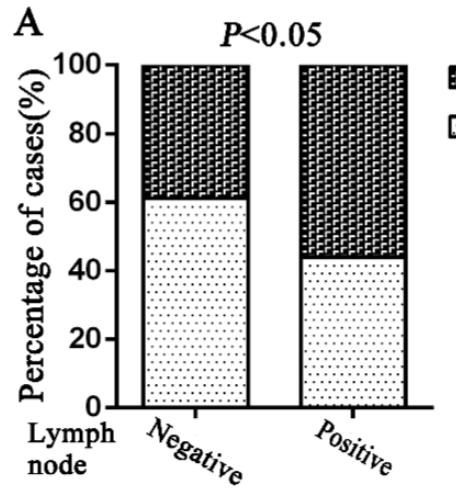

C

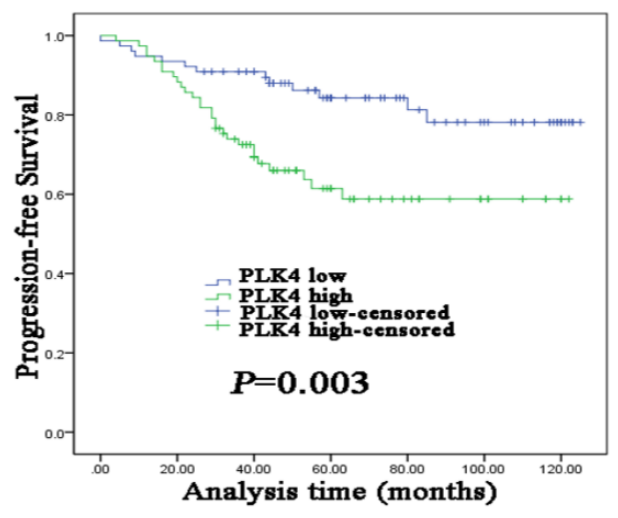

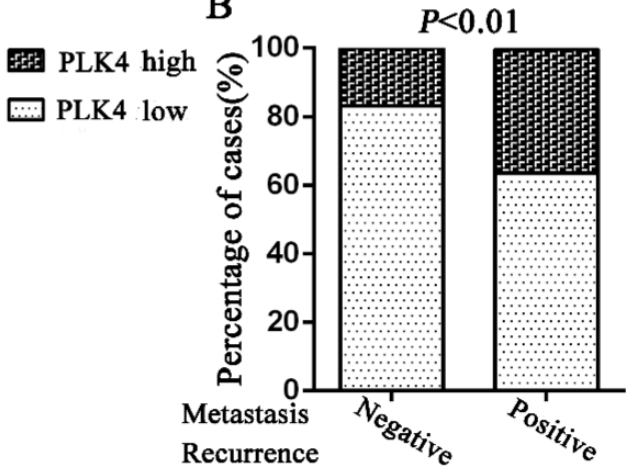

$\mathrm{D}$

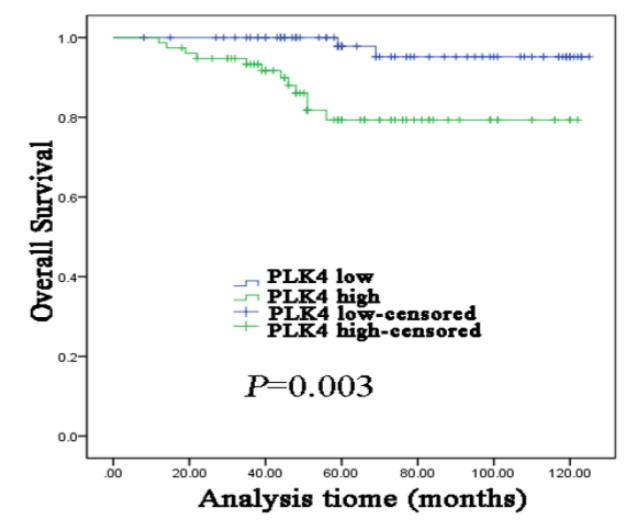

Figure 3. PLK4 overexpression indicates high risk of lymph node metastasis and distant metastasis or surrounding recurrence, and poor prognosis in breast cancer. Lymph node status (A) and metastasis or recurrence status (B) was analyzed based on the PLK4 expression which was divided into low or high by the median values. PFS (C) and OS (D) curves were generated based on the PLK4 protein expression statuses in 154breast cancer samples. 

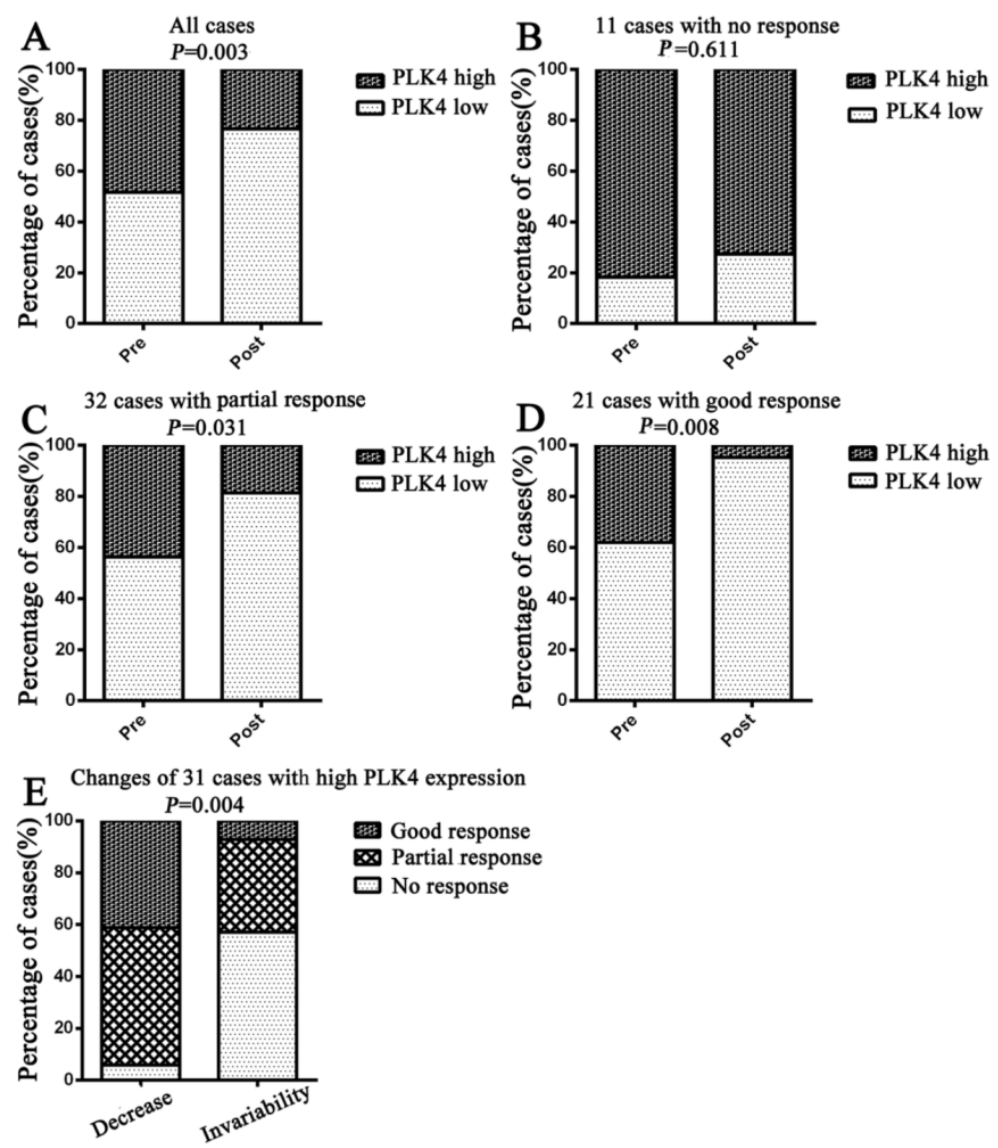

Figure 4. Changes of PLK4 expression before and after taxane-based neoadjuvant chemotherapy. Changes of the percentage of low or high PLK4 expression were examined in all cases(A), cases with no response(B), cases with partial response(C) and cases with good response(D). Cases with high PLK4 expression before neoadjuvant chemotherapy were particularly analyzed in $(E)$.

\section{Discussion}

The functions of PLK4 identified by current date are related to its fundamental role in diverse mitotic events, including centrosomes duplication, cell spreading, motility, and cytokinesis [17-21]. In early G1 phase, PLK4 regulates centriole duplication through autophosphorylation, while limits centriole duplication to once per cycle through degradation of PLK4 by a negative feedback loop [22, 23]. PLK4 initiates and catalyzes centriole duplication and elongation by interacting with the cyclin-dependent kinase CDK2, CP110, and the PLK4 downstream regulator spindle assembly 6 homolog (SAS6) throughout $S$ phase [19]. However, the factors regulating the PLK4 gene expression have not been fully understood. Some studies have reported that SAPK pathways and P53 cooperatively suppress PLK4 activity [24-26], and the two pathways are frequently inactive in malignant tumors. NFKB controls the expression of PLK4, and PLK4 may be a new NFKB target gene, providing a direct link between NFKB and centrosome duplication [27].

PLK4 expression status has been previously reported in colorectal cancer [5], gastric cancer [2], and hepatocellular carcinoma [6]. However, PLK4 expression status was different among these kinds of cancers. PLK4 overexpressed in colorectal cancer and gastric cancer, while had low-expression levels in hepatocellular carcinoma. Some reports has demonstrated that PLK4 is highly expressed in breast cancer tissues or cells $[28,29]$, but the role of PLK4 in breast cancer has not been thoroughly elucidated.

In the present study, we investigated the PLK4 expression status and its prognostic value in breast cancer, and determined the relationship of PLK4 expression with clinicopathological parameters and response to taxane-based neoadjuvant chemotherapy of breast cancer patients. Results indicated that the expression of PLK4 mRNA was commonly upregulated in breast cancer, and PLK4 was highly expressed in breast cancer tissues and significantly associated with lymph node metastasis and distant metastasis or surrounding recurrence. In addition, Kaplan-Meier survival analysis showed that high expression PLK4 was an unfavorable prognostic factor by both PFS and OS. Moreover, PLK4 expression status was identified as a risk factor for 
both PFS and OS in Univariate Cox-regression analysis, while only for PFS in Multivariate Cox-regression analysis. Our results suggested that PLK4 might play an important role in breast cancer invasion and metastasis, and PLK4 could be a potential prognostic factor of breast cancer.

Even though there is a scarcity of data investigating the putative role of PLK4 in breast cancer; we still could find evidence to support its contribution to breast cancer biology. Jacqueline et al [29] demonstrated that PLK4 mRNA levels were significantly higher in breast cancer cells than their normal tissue counterparts by using a quantitative RT-PCR, and PLK4 was overexpressed in $26 \%$ of all breast cancer tumors by examining the microarray of breast cancer. In addition, they found breast cancer cells might be predominantly dependent on PLK4 for survival [29]. Rosario et al. revealed that PLK4 facilitated breast cancer cells migration, and their findings may promote an association between increased PLK4 expression, cancer progression and death from metastasis in breast cancer patients [20]. In addition, PLK4-interacting proteins may also support this point. For example, Ect2, binding and phosphorylating by PLK4, is a component of the Mamma Print genetic signature that predicts the recurrence risk of breast cancer [21]. In short, despite the different adopted methodologies, the above studies' data are consistent with ours.

Furthermore, we investigated the response of PLK4 in taxane-based neoadjuvant chemotherapy for the first time, and found that PLK4 expression was a negative predictor of response to taxane-based neoadjuvant chemotherapy. Taxanes such as paclitaxel and docetaxel highly interfere with microtubule functions and have been used in many malignant tumors. While taxanes treated a variety of tumors successfully, some patients did not benefit from this treatment or even insensitive to further treatment. Although the mechanisms behind the insensitivity of tumor cells to taxanes in these patients is not well understood, cell culture studies have identified some $\alpha$-tubulin and $\beta$-tubulin mutations which contribute to the resistance to taxanes [30,31]. $\mathrm{\gamma}$-Tubulin is responsible for microtubule nucleation by forming a multiprotein ring complex $(\gamma$-TuRC) [11]. PLK4 is an upstream regulator for $\gamma$-tubulin and dependent on formatting of the $\gamma$-tubulin-containing structure [12]. Our results indicated that the PLK4- $\gamma$-tubulin axis maybe a novel mechanism for the resistance to taxanes which needs further investigation.

Indeed, we did not find the changes of PLK4 expression between the biopsy and tumor specimens of breast cancer patients with no response to neoadjuvant chemotherapy were not found, however, it was significantly changed in the patients with partial response or good response. It has been reported that inhibitors of PLK4 expression might be a potential anticancer agents [32]. Thus, targeting PLK4 with inhibitors could be used in breast cancer therapy, and may overcome the resistance to taxane-based chemotherapy, especially when PLK4 was highly expressed in the breast cancer.

In summary, we demonstrated that overexpression of PLK4 may playan important role in breast cancer invasion and metastasis, and PLK4 could be a potential prognostic factor of breast cancer. Moreover, PLK4 inhibitor may function as a potential anticancer agent, and overcome the resistance to taxane-based chemotherapy for breast cancers.

\section{Acknowledgments}

We thank Lanjin Zhang (Gastrointestinal and Liver Pathology, University Medical Center of Princeton at Plainsboro, Rutgers University, Plainsboro, USA) for expert comments. This work was supported by grants from the National Natural Science Foundation of China (Grant No.30930038, 81202101).

\section{Conflict of interest}

The authors declare that they have no conflict of interest.

\section{References}

1. van de Weerdt BCM, Medema RH. Polo-like kinases - A team in control of the division. Cell Cycle. 2006; 5: 853-64.

2. Shinmura K, Kurabe N, Goto M, Yamada H, Natsume H, Konno H, et al. PLK4 overexpression and its effect on centrosome regulation and chromosome stability in human gastric cancer. Mol Biol Rep. 2014; 41: 6635-44.

3. Ganem NJ, Godinho SA, Pellman D. A mechanism linking extra centrosomes to chromosomal instability. Nature. 2009; 460: 278-U146.

4. Bettencourt-Dias M, Hildebrandt F, Pellman D, Woods G, Godinho SA. Centrosomes and cilia in human disease. Trends Genet. 2011; 27: 307-15.

5. Macmillan JC, Hudson JW, Bull S, Dennis JW, Swallow CJ. Comparative expression of the mitotic regulators SAK and PLK in colorectal cancer. Ann Surg Oncol. 2001; 8: 729-40.

6. Liu LL, Zhang CZ, Cai MY, Fu J, Chen GG, Yun JP. Downregulation of Polo-Like Kinase 4 in Hepatocellular Carcinoma Associates with Poor Prognosis. Plos One. 2012; 7.

7. Chia S, Swain SM, Byrd DR, Mankoff DA. Locally advanced and inflammatory breast cancer. J Clin Oncol. 2008; 26: 786-90.

8. Gogia A, Raina V, Deo SV, Shukla NK, Mohanti BK, Sharma DN. Taxane and anthracycline based neoadjuvant chemotherapy for locally advanced breast cancer: institutional experience. Asian Pacific journal of cancer prevention : APJCP. 2014; 15: 1989-92.

9. Chanat C, Delbaldo C, Denis J, Bocaccio F, Cojean-Zelek I, Le Guyader N. Dose intensity and toxicity associated with Taxotere formulation: a retrospective study in a population of breast cancer patients treated with docetaxel as an adjuvant or neoadjuvant chemotherapy. Anti-cancer drugs. 2015; 26: 984-9.

10. Schott AF, Hayes DF. Defining the Benefits of Neoadjuvant Chemotherapy for Breast Cancer. J Clin Oncol. 2012; 30: 1747-9.

11. Moritz M, Agard DA. gamma-Tubulin complexes and microtubule nucleation. Curr Opin Struc Biol. 2001; 11: 174-81.

12. Kuriyama R, Bettencourt-Dias M, Hoffmann I, Arnold M, Sandvig L. gamma-Tubulin-containing abnormal centrioles are induced by insufficient Plk4 in human HCT116 colorectal cancer cells. J Cell Sci. 2009; 122: 2014-23.

13. Elston CW, Ellis IO. Pathological prognostic factors in breast cancer. I. The value of histological grade in breast cancer: experience from a large study with long-term follow-up. Histopathology. 1991; 19: 403-10. 
14. Liu FF, Lang RG, Zhao J, Zhang XM, Pringle GA, Fan Y, et al. CD8(+) cytotoxic $\mathrm{T}$ cell and FOXP3(+) regulatory $\mathrm{T}$ cell infiltration in relation to breast cancer survival and molecular subtypes. Breast Cancer Res Tr. 2011; 130: 645-55.

15. Liu FF, Li YQ, Ren MJ, Zhang XM, Guo XJ, Lang RG, et al. Peritumoral FOXP3(+) regulatory $\mathrm{T}$ cell is sensitive to chemotherapy while intratumoral FOXP3(+) regulatory $\mathrm{T}$ cell is prognostic predictor of breast cancer patients. Breast Cancer Res Tr. 2012; 135: 459-67.

16. Ogston KN, Miller ID, Payne S, Hutcheon AW, Sarkar TK, Smith I, et al. A new histological grading system to assess response of breast cancers to primary chemotherapy: prognostic significance and survival. Breast. 2003; 12: 320-7.

17. Cunha-Ferreira I, Rodrigues-Martins A, Bento I, Riparbelli M, Zhang W, Laue E, et al. The SCF/Slimb Ubiquitin Ligase Limits Centrosome Amplification through Degradation of SAK/PLK4. Curr Biol. 2009; 19: 43-9.

18. Guderian G, Westendorf J, Uldschmid A, Nigg EA. Plk4 trans-autophosphorylation regulates centriole number by controlling beta TrCP-mediated degradation. J Cell Sci. 2010; 123: 2163-9.

19. Habedanck R, Stierhof YD, Wilkinson CJ, Nigg EA. The Polo kinase Plk4 functions in centriole duplication. Nat Cell Biol. 2005; 7: 1140-6.

20. Rosario CO, Kazazian K, Zih FSW, Brashavitskaya O, Haffani Y, Xu RSZ, et al. A novel role for Plk4 in regulating cell spreading and motility. Oncogene. 2015; 34: 3441-51.

21. Rosario CO, Ko MA, Haffani YZ, Gladdy RA, Paderova J, Pollett A, et al. Plk4 is required for cytokinesis and maintenance of chromosomal stability. P Natl Acad Sci USA. 2010; 107: 6888-93.

22. Holland AJ, Lan WJ, Niessen S, Hoover H, Cleveland DW. Polo-like kinase 4 kinase activity limits centrosome overduplication by autoregulating its own stability. J Cell Biol. 2010; 188: 191-8.

23. Sillibourne JE, Tack F, Vloemans N, Boeckx A, Thambirajah S, Bonnet P, et al. Autophosphorylation of Polo-like Kinase 4 and Its Role in Centriole Duplication. Mol Biol Cell. 2010; 21: 547-61.

24. Kurinna S, Stratton SA, Coban Z, Schumacher JM, Grompe M, Duncan AW, et al. p53 Regulates a Mitotic Transcription Program and Determines Ploidy in Normal Mouse Liver. Hepatology. 2013; 57: 2004-13.

25. Nakamura T, Saito $H$, Takekawa M. SAPK pathways and p53 cooperatively regulate PLK4 activity and centrosome integrity under stress. Nat Commun. $2013 ; 4$.

26. Li J, Tan MJ, Li L, Pamarthy D, Lawrence TS, Sun Y. SAK, a new polo-like kinase, is transcriptionally repressed by p53 and induces apoptosis upon RNAi silencing. Neoplasia. 2005; 7: 312-23.

27. Ledoux AC, Sellier H, Gillies K, Iannetti A, James J, Perkins ND. NF kappa B regulates expression of Polo-like kinase 4. Cell Cycle. 2013; 12: 3052-62.

28. Marina M, Saavedra HI. Nek2 and Plk4: prognostic markers, drivers of breast tumorigenesis and drug resistance. Front Biosci-Landmrk. 2014; 19: 352-65.

29. Mason JM, Lin DCC, Wei X, Che Y, Yao Y, Kiarash R, et al. Functional Characterization of CFI-400945, a Polo-like Kinase 4 Inhibitor, as a Potential Anticancer Agent. Cancer Cell. 2014; 26: 163-76.

30. Yin SH, Zeng CQ, Hari M, Cabral F. Random Mutagenesis of beta-Tubulin Defines a Set of Dispersed Mutations That Confer Paclitaxel Resistance. Pharm Res-Dordr. 2012; 29: 2994-3006.

31. Yin SH, Zeng CQ, Hari M, Cabral F. Paclitaxel Resistance by Random Mutagenesis of alpha-Tubulin. Cytoskeleton. 2013; 70: 849-62.

32. Sampson PB, Liu Y, Patel NK, Feher M, Forrest B, Li SW, et al. The Discovery of Polo-Like Kinase 4 Inhibitors: Design and Optimization of Spiro[cyclopropane-1,3 '[3H]indol]-2 '(1 ' H)-ones as Orally Bioavailable Antitumor Agents. J Med Chem. 2015; 58: 130-46. 